\title{
Coloration of Polyester Fibers for Securities Protection from Counterfeit
}

\author{
V.A. Goldade ${ }^{1,2, *}$, N.V. Kuzmenkova ${ }^{3}$ and V.E. Sytsko ${ }^{3}$ \\ ${ }^{1}$ Francisk Skorina Gomel State University, Gomel, Belarus \\ ${ }^{2}$ V.A. Belyi Metal-Polymer Research Institute of National Academy of Sciences of Belarus, Belarus \\ ${ }^{3}$ Belarusian Trade and Economics University of Consumer Cooperatives, Gomel, Belarus
}

\begin{abstract}
The results of modifying polyester fibers with luminescent colorants by crazing mechanism have been given. By means of REM and AFM stages of crazes initiation, their transformation into fibrillar structure of fiber and redistribution of modifiers incorporated into crazes in the surface layer of fiber have been revealed. Techniques of concentrating luminescent colorants on local sections of fiber with alternation of unprocessed segments and segments containing target additives have been described. Polyester fibers with alternating by length sections of luminescent division of colors have been obtained. It was shown that effective fiber coloration takes place when the drawing out degree $\varepsilon^{*}$ is between 2 and 3 . Fibers modified by Oxazine and Rhodamine colorants are characterized by highest intensity of luminescence in UV radiation. The conclusion has been made that technology of modifying chemical fibers by cazing mechanism allowing implementing measured incorporation of target additives on local sections of fiber satisfy in the optimal way the protection criteria of paper filled with such fibers from counterfeiting.
\end{abstract}

Keywords: Chemical fibers, modification, crazing, protection, colorants, luminescence.

\section{INTRODUCTION}

A crucial issue for state economic safety has been prevention of securities (banknotes, checks, bills, tickets, other documents and securities) from counterfeiting. It is especially urgent under conditions of intensifying development and wide implementation of sophisticated technologies of material processing. Manufacturing a security or a bank note is supposed to protect all its elements - paper substrate, paints, printing techniques, images - with special methods [1]. Document protection system has been embedded during production process, starting with developing original layout, choice of technological process, materials for manufacturing paper cloth, printing techniques, etc. In world practice multi-level protection systems have been used. They include such sets of methods and facilities which stipulate inexpediency of securities counterfeiting [2].

Paper cloth being the base of printing production, its protection is considered to be the most significant measure to prevent securities falsification. Protection facilities embedded in paper itself incorporate water marks, special types of fibers and threads, chads, chemical reactive additives et al. [3]. The advantages of such decision are simplicity of paper identification and complexity of manufacturing an imitation close to the original.

*Address correspondence to this author at the Francisk Skorina Gomel State University, 104 Sovetskaja St., 246019 Gomel, Belarus; Tel: +375232774656; Fax: +375232774808; E-mail: victor.goldade@gmail.com
The aim of the present paper is: i) to make a choice of methodology of modifying polyester fibers to protect securities, and ii) to investigate the luminescent properties of polyester fibers modified with different colorants during orientation stretching by crazing mechanism.

\section{CHEMICAL FIBERS FOR SECURITIES AND THEIR REQUIREMENTS}

Protective fibers represent short sections of fiber up to $10 \mathrm{~mm}$ length. They are incorporated into paper substrate during its manufacturing and can be chaotically distributed in cellulose pulp or placed as stripes of 3-4 cm wide [4]. Chemical fibers are imparted particular properties which allow implementing additional elements of protection. Fibers can be achromatic or colored with various range of colors. Fibers processed by luminescent coloring agents enhance the degree of protection of documents significantly. Luminescent fibers both achromatic and colored ones are manufactured at daylight illumination, achromatic fibers ensuring more reliable protection. If protective fibers incorporated into document paper substrate are of different colors, it is considered enough to add fibers of three types differing by color of fiber at daylight illumination or by color of its glow when affected by ultraviolet (UV) radiation. It is not desirable to use fibers with blue color glow since they are easier to falsify [3]. Protective fibers with discontinuous coloring are the most effective ones, for instance, twocolored fibers, which look single-colored externally, but under a magnifier the alternation of colors is noticeable. 
Profiled protective fibers are distinguished by a specific form of cross-section obtained in the process of shaping. They are easily identified, have relatively low cost, are impossible to be imitated by printing or other methods. Experts of public enterprise "Goznak" of the Russian Federation developed so-called phased fibers ("Zone" fibers) with variable diameter, where segments with different cross-section form alternate. Such kind of fiber can have alternating dyed and undyed, luminescent and UV-passive segments [4].

There have been developed and applied bicomponent fibers having different optical properties in crosswise direction. Such fibers are formed from composition melts of limitedly compatible or incompatible polymers (copolymers) [5]. To enhance the degree of protection, fibers with additional special properties are produced, for instance, by attaching ferromagnetic properties to them [4].

Protective fibers must possess the following properties:

- Visible luminescence controlled visually or with special devices (ultra-violet, infra-red radiation, et al.)

- $\quad$ have optimal length $(3-6 \mathrm{~mm})$;

- $\quad$ thickness of a separate filament must not exceed thickness of paper it is used to protect;

- be thermal-resistant at temperatures up to140 ${ }^{\circ} \mathrm{C}$.

Properties of chemical fibers determining the degree of protection of securities, first of all, the reliability of fixing protective elements on fiber, are formed in the process of manufacturing and target modifying of fibers.

\section{TRADITIONAL METHODS OF COLORATION CHEMICAL FIBERS}

The classical method of obtaining colored chemical fibers is to fill polymer melt or solution with disperse dye particles. Black color fibers are normally made by this method. To obtain fibers of other colors, as a rule, organic dyes are used. The range of the latter is quite narrow, and monochrome fibers obtained with them are distinguished by inconspicuous coloring and have restricted efficiency as a means of protection of documents from counterfeiting [6].

The method of dyeing polyester fibers when they are kept in liquid dyeing bath at temperatures $50-55^{\circ} \mathrm{C}$ for 2-5 minutes is widely spread. In the result of this rather long process fibers also acquire inconspicuous coloring. The advanced variant of this method suggesting treatment of just-formed threads in aqueous dyeing bath in two stages at heating up to temperatures 100 and $105{ }^{\circ} \mathrm{C}$ is more productive. At the first stage coloration is performed at constant moderate speed of drawing threads in bath, at the second one speed is increased by 1.5-10 times. The method is supposed to produce a moderate thread tension and provide even distribution of dye along the fiber.

There is a known method of dyeing by liquid colorant being deposited on fiber surface by atomizer spraying under pressure. Dye is distributed on fiber at random, it being rather difficult to control its amount and coloring shade [7].

Dyeing fiber on local segments allows enhancing the degree of protection considerably. One of the methods of such dyeing suggests producing twist yarn from fiber to be dyed first and then cut into short sections [8]. Staple fiber obtained by this way has alternating dyed and undyed sections and may be repeatedly dyed with the other paint. The disadvantages of this method are labor input, lack of clear edges between sections and impossibility to control the length of dyed segments.

At discrete dyeing of fibers coloring agent and activating liquid are used. First, separate segments of fibers are charged unipolarly by depositing activating liquid drops on them. Then fibers with charged and uncharged segments are placed into coloring agent, and sections of different intensity coloring are obtained. Flexible control of dyeing is performed by computer. This allows automating dyeing process and dispensing activating liquid drops accurately [7].

Dyeing chemical fibers with dye vapors is a comparatively new method [9]. Vapor-phase technology of dyeing is performed by transferring dye into a sublimated state, from which dye vapor is adsorbed by fiber surface. The intensity of coloring depends on speed of drawing filament through dyeing chamber (at lower speed intensity is higher).

The above methods of dyeing provide depositing dye on fibers surface due to which durability of coloring to a great extent depends on the degree of thermodynamic or technological affinity of dye with fiber material. 


\section{CHEMICAL FIBERS DYED BY CRAZING MECHANISM}

A prospective methodology of modifying polymer chemical fibers is incorporation of target additives into structure of fiber by crazing mechanism. Crazing mechanism of polymers has been described in the earlier works [10-14].

Polymer crazing takes place in the process of orientation drawing carried out in liquid adsorptionactive medium [11]. There may be marked out several stages in it. At the first stages of stretching, crazes (micro cracks, their walls being connected by fibrillar strains) are generated on fiber surface. On further deformation crazes grow in the direction perpendicular to the axis of elongation of a sample until they intersect its cross-section [12]. At the next stage crazes widen in the direction of the axis of elongation, and fiber turns into oriented fibrillar state. In liquid technological medium arising crazes are instantly filled with fluid phase [14] which makes it possible to incorporate practically any incompatible additives (bactericides, fire retardants, coloring matters, et al.) into polymer structure providing their even distribution in the surface layer of fibers [10]. Additives captured by crazes are held in the superficial layer of fiber $[12,13]$.

Figure 1a and $\mathbf{b}$ shows micrographs of fiber, stretched in surface-active liquid. In area I crazes are initiated, in area II crazes widen and neck is formed on fiber. Figure 1b shows enlarged image of one of the crazes.

One can see that walls (1) of crack (2) are connected by strains (3). The scheme of this structural formation is given in Figure 1c [13]. Further drawing of fiber is accompanied by gradual transition of fiber material into fibrillar structure of strains. In area IV superficial layer of fiber mainly consists of parallel fibrils.

The most intensive saturation of crazes with modifying liquid occurs in areas II and III at the step of neck forming. In area IV captured liquid is redistributed in superficial layer of fiber [15].

At dyeing by crazing mechanism, fiber is exposed to drawing in surface-active liquid medium containing coloring matter. Such technology allows giving stable coloring to achromatophilous polyester fibers, it being highly productive and easily fitting in the standard process of manufacturing chemical fibers without requiring considerable modification of technological equipment [16].

The structure of fibers exposed to dyeing in the process of crazing has been investigated by the method of atomic-force microscopy (AFM) with NT-206 device ('Microtestmachine' Co, Belarus).

Figure 2a shows a 'slammed' craze profile, its depth being about $0.2 \mu \mathrm{m}$. It does not mean that coloring agent penetrates exactly to such depth. Figure $\mathbf{2 b}$ demonstrates REM-image of a lateral shear of dyed fiber where one can see that the depth of penetration of luminescent coloring agent (it luminesces under electron beam impact) is at least two degrees higher.

One can imagine that coloring agent penetrates to the whole depth of an opening craze $(200 \div 250 \mu \mathrm{m})$ and even deeper - at the expense of diffusion. In the process of relaxation of stretching strains in fiber (zone IV in Figure 1a) craze walls clamp. Particles penetrated into crazes do not let the walls interlock completely. Craze profile in Figure $\mathbf{2 a}$ fits just this situation.

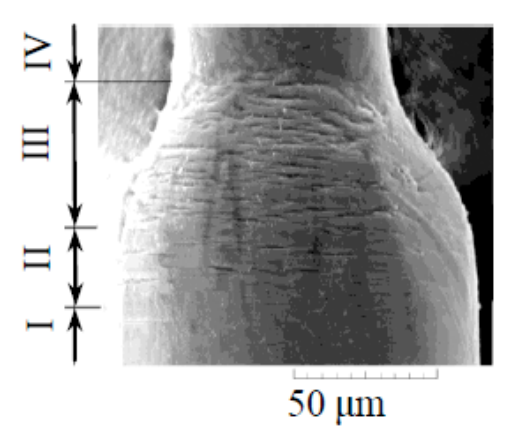

$a$

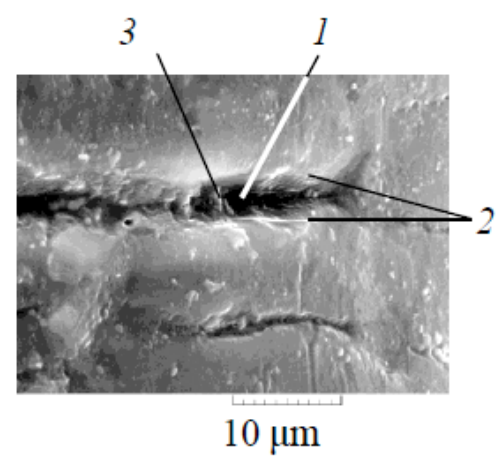

$b$

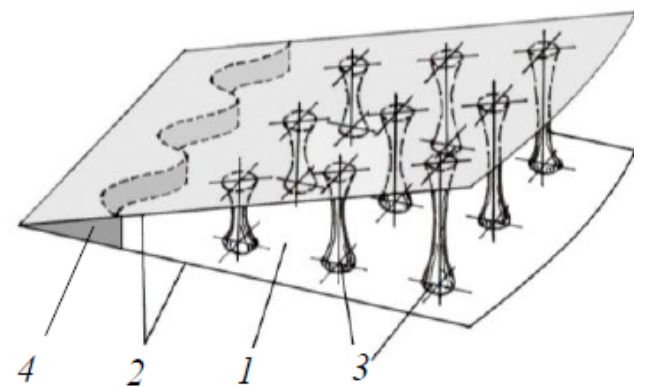

C

Figure 1: REM-images $(a, b)$ and scheme $(c)$ of crazes forming in polyethylene terephthalate (PET) fibers. Roman numerals indicate areas of strained state of fiber where occur: I - initiation of crazes, II - growth, III - widening of crazes, IV - formation of fibrillar structure of fiber. 1 - crack walls, 2 - crack, 3 - strains, 4 - fiber's material. 


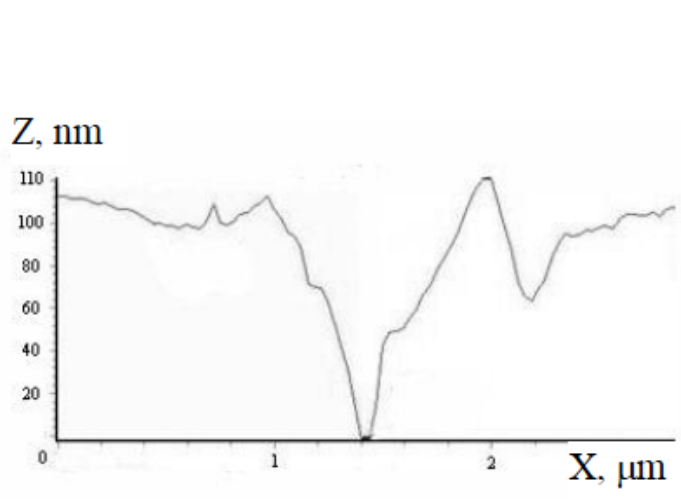

$a$

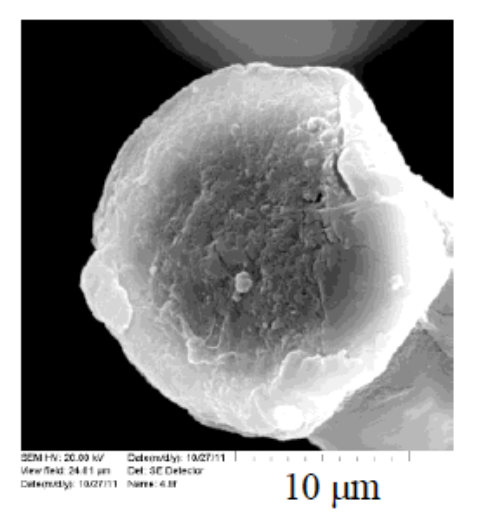

$b$

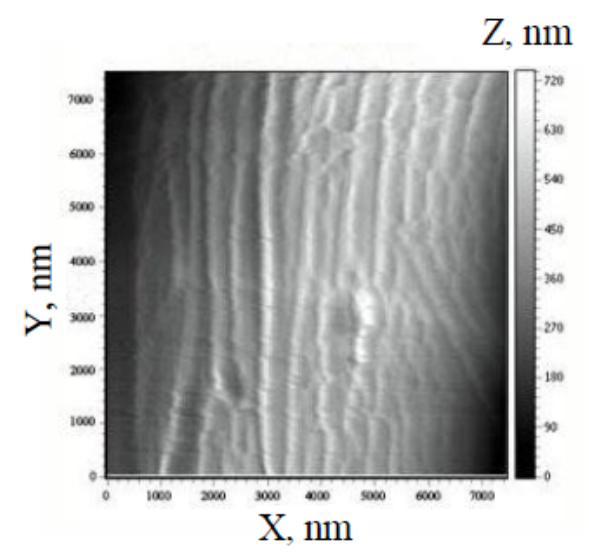

$c$

Figure 2: Structure of PET fiber dyed by Rhodamine colouring agent: $a$ - craze profile, $b-$ lateral shear of fiber, $c-$ fiber surface.

Fibrillisation of superficial layer of fibers at stretching (stretching ratio $\varepsilon^{*} \geq 3.0$ ) causes fibrillar formations yielding on fiber surface and forming specific corrugations-shaped texture, registered in Figure $2 c$ by AFM method.

Crazing allows combining dyeing with incorporating any modifying additives to give additional useful properties to fiber (ferromagnetism, capability to absorb radiation, et al.) This gives broad possibilities to further improving technology of modifying fibers, in particular, to dyeing fibers in areas alternating with undyed ones and appears to be the most prospective way for obtaining fibers by realizing various mechanisms of protection of securities.

\section{DISCONTINUOUS COLORATION OF FIBERS}

For discontinuous modifying of chemical fibers by crazing mechanism an original device has been used [17]. It contains 2 sets of withdrawal rollers for transporting and stretching of fibers, the first set's of rollers rotation speed being lower than the other's one. The last roller of the first set has been made toothed with teeth spacing equal to the length of dyed fiber segment. The contact zone of toothed roller with counterbody, which is a conjugated smooth support plate, is in the bath and is dipped into surface-active liquid containing coloring matter. The bath bottom is coated with elastic covering.

The drawing point of modified fiber, at which generation of crazes starts under proper degree of drawing and dampening by surface-active liquid, is at the exit of fiber from "toothed roller/ counterbody" contact (Figure 3). When fiber is pressed to counterbody tooth by force $F_{1}(a)$, the degree of drawing is maximal and sufficient for initiation of crazes, therefore fiber segment at exit from contact is dyed due to capturing coloring agent by crazes. When slipping along counterbody, fiber is between teeth $(b$, force $F_{0}$ ), the degree of its drawing is lower than it is necessary for craze initiation. As a result, fiber segment between positions where fiber was pressed to counterbody by adjacent teeth remains undyed.
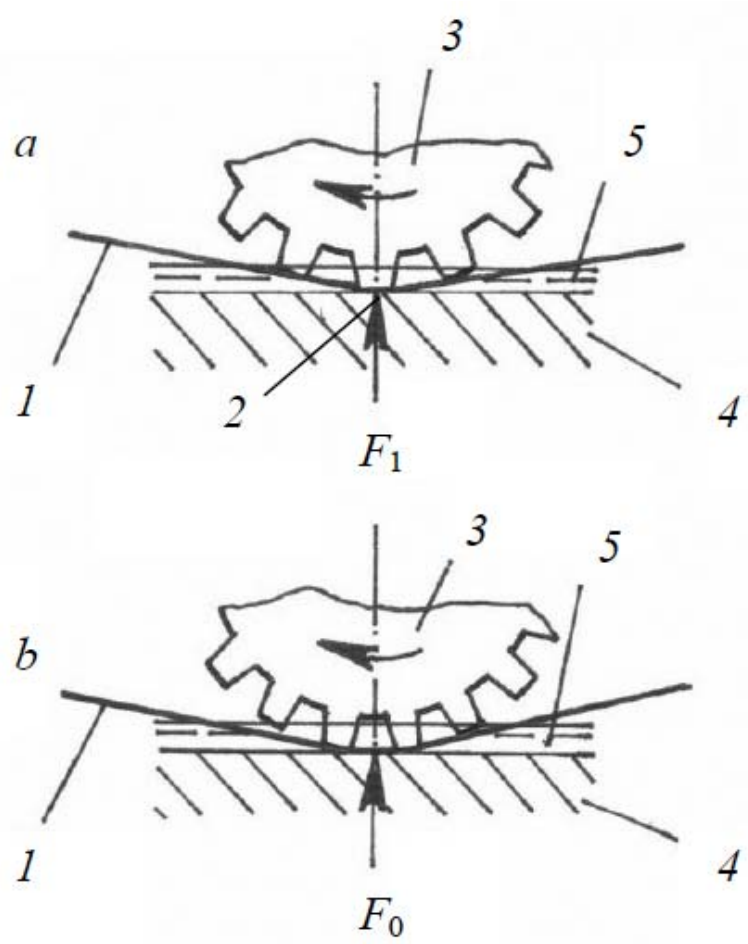

Figure 3: Scheme of interaction of fiber and toothed roller at discrete modifying: $a$ - fiber is pressed to bath bottom, $b-$ fiber is between teeth. 1 - fiber, 2 - drawing point, 3 toothed roller, 4 - bath bottom, 5-surface-active liquid.

The experiments were carried out with non-oriented PET fibers of nominal linear density 0.33 tex mark $A$ of 
'Mogilevkhimvolokno' Co. (Belarus) production. To treat the fibers, solutions in isopropyl alcohol (propanol-2) luminescent coloring agents - Phenalemine-160, Oxazine-17, Rhodamine-C, Fluorescein, Eosin- $\mathrm{H}$ and EK-3FP mark colorant - have been used. Dyed fibers have been investigated in UV-light with "Peleng VK-04" designed to control documentary stamps (included in Belarus Public Register of Equipment to check identity of securities and documents).

It has been determined that at stretching ratio $\varepsilon^{*}=$ 2.5 (corresponds to specific elongation $\varepsilon=250 \%$ ) fibers modified by Phenalemine solution have the lowest glow intensity. The effect of polarization division of luminescence in such fiber is near to similar indicator of original fiber. Other coloring agents noticeably exceed phenalemine by glow brightness. Oxazine is distinguished by high brightness. Fibers dyed with it which are practically clear in visible light acquire coloring in UV-light (Figure 4). EK-3 FP colorant causes the most intense glow in UV radiation.

In the result of by-turn modifying by two fluorescent coloring agents, fiber with the effect of alternating polarization division of colors has been obtained: areas of orange-pink and purple colors. Thereto fiber was first evenly dyed (at $\varepsilon^{*} \approx 2$ ) by EK-3FP coloring agent solution, then exposed to discontinuous dyeing (at $\varepsilon^{*}=$ $2.7 \div 3.0$ ) by Oxazine solution. Choosing combinations of coloring agents differing by glow color in UV radiation it is possible to give fibers set alternation of colors implementing a planned scheme of protecting paper filled with fiber bits.

\section{LUMINESCENCE OF DYED FIBERS}

Luminescence spectra have been obtained with a space-saving spectrometer with the CCD-ruler method of recording. With small overall dimensions the device enables $0.3 \mathrm{~nm}$ resolving ability and has sensitivity making it possible to register luminescence spectra of organic dyes solutions in concentrations up to $10^{-10}$ mole per liter. The effective range of the spectrometer is $400 \div 820 \mathrm{~nm}$. The excitation source of luminescence spectra is either $405 \mathrm{~nm}$ wavelength semiconductor laser or $37 \mathrm{~nm}$ wavelength gas laser. The spectrometer is run by the computer and has simple and handy software.

The main units of the appliance are optical system, CCD camera photo-receiving component and camera and computer information input control board.

ILX-511 CCD ruler (Sony) has been employed as photo detector. It consists of 2048 elements, their size being $14 \times 200$ micron. Modes of operation are defined by the program, accumulation time being from $10 \mathrm{~ms}$ до $50 \mathrm{~s}$.

Diffusely scattered radiation from light source is coming through a body entrance slit into a collimator lens where it is transformed into parallel light beam. The received beam is reflected by a deflecting mirror, goes through the cuvette with material under investigation or fixed fiber bundle, then it is brought together by camera lens and supplied onto CCD camera photo-receiving component. Inside the spectrometer's body under bottom plate there is the board by which camera and computer information input control is exercised.

To approximate data characterizing luminescence spectra of dye solutions, the least-squares method which makes it possible to achieve high precision approximation and Fourier filtration have been used. The latter has been widely employed by spectral analysis for cleaning signal from noise and smoothing spectrum. Data processing has been performed with Statistica v.6.1 and OriginPro v.7.0 programs.

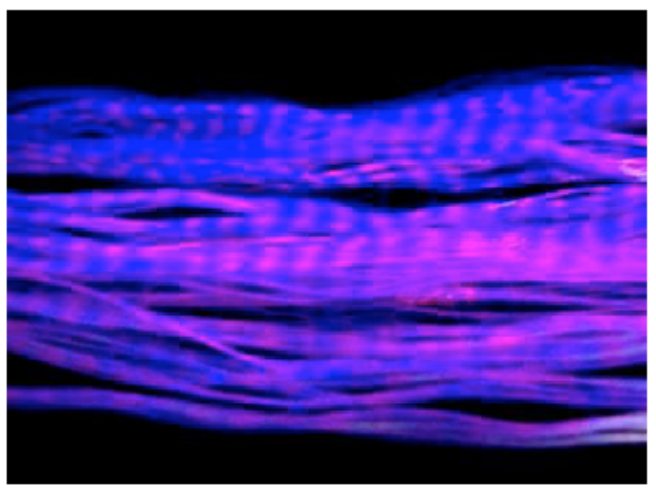

Figure 4: The view of PET fibers discretely dyed by Oxazine: $a$ - in visible light, $b$ - in UV-light. 
The degree (ratio) of fiber drawing affects modifying substance sorption [15]. Fiber deformation on drawing from 50 to $250 \%$ leads to increasing the amount of dye captured by crazes. On reaching the degree of drawing $\varepsilon^{*}=3.0 \div 3.5$ (i.e. $\varepsilon=300 \div 350 \%$ ), when crazes collapsing and transition from loose fiber structure to tight one starts, the inverse process begins - dissolving agent is squeezed out of crazes. Distances between fiber fibrils in the process of drawing are continually decreasing and eventually become comparable with the sizes of dye molecules. As a result, peculiar ultrafiltration of dye solution occurs at molecular level which leads to dye molecules being captured by fiber structure, mainly pure dissolvent being filtered into a bath. To obtain totally oriented sample, the degree of drawing must be $3.8 \div 4.5$ [16].

Thus, more intensive craze formation takes place at drawing ratio $\varepsilon^{*}$ within 2 and 3 . At drawing ratio $\varepsilon^{*}<2$ fiber surface does not capture modifying substance in sufficient amount while at reaching drawing ratio $\varepsilon^{*} \approx 3$ crazes are filled with modifying particles at most and adsorption of a large quantity of particles is impossible.

To investigate dye concentration in modifying liquid impact on luminescence intensity, spectral characteristics of Rhodamine solutions at $0.05 \div 4 \mathrm{~g} / \mathrm{l}$ dye concentration and fibers modified by it have been studied. As dye concentration is increased, not only luminescence intensity of the solutions changes, but also their spectral characteristics (Figure 5). Composition with Rhodamine concentration $0.1 \mathrm{~g} / \mathrm{l}$

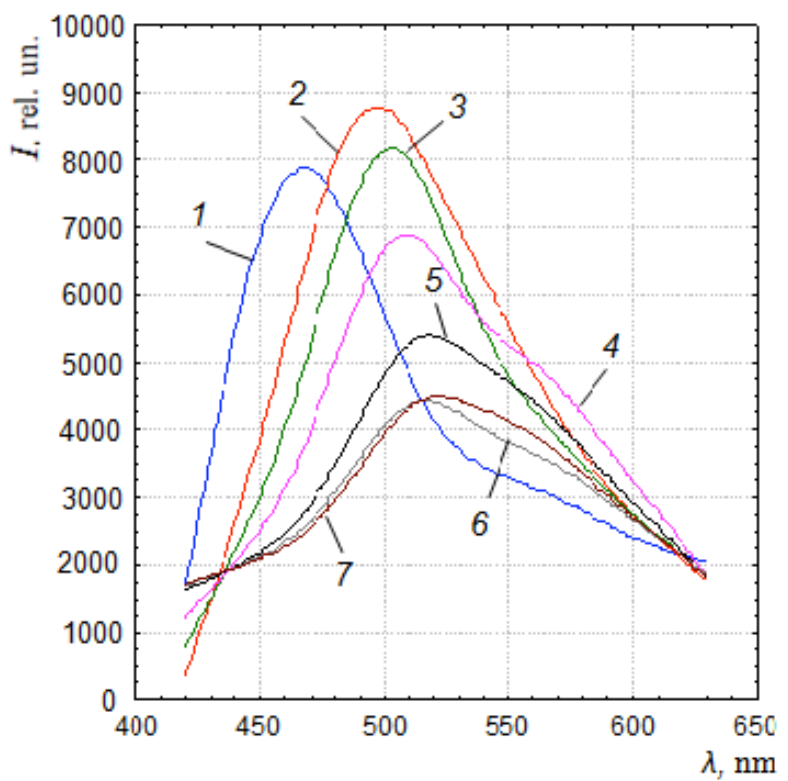

Figure 5: Luminescence of Rhodamine solutions in propanol at dye concentration: $1-0.05 \mathrm{~g} / \mathrm{l} ; 2-0.1 \mathrm{~g} / \mathrm{l} ; 3-0.2 \mathrm{~g} / \mathrm{l} ; 4$ $0.5 \mathrm{~g} / \mathrm{l} ; 5-1 \mathrm{~g} / \mathrm{l} ; 6-2 \mathrm{~g} / \mathrm{l} ; 7-4 \mathrm{~g} / \mathrm{l}$. (spectrum 2) possesses the most intensive luminescence. With further dye concentration increasing, concentration quenching takes place and luminescence maximum shifts to long-wave spectral region. Luminescence intensity is low and changes insignificantly at dye concentration higher than $1 \mathrm{~g} / \mathrm{l}$.

When modifying with luminescent dyes as dye concentration grows in the solution, fibers luminescence color changes from pink to reddishorange. At the same concentration of dye in the solution, luminescence spectra of dyed fibers (Figure 6) differ markedly from luminescence spectra of dye solutions (Figure 5). The reasons of differences between spectra have been different aggregative states and light refraction coefficients of luminescent media. Besides, the difference between spectra of dye solutions and luminescent fibers, in our opinion, is stipulated by the presence of the original fiber's proper luminescence. One can assume that when treating fiber with dye solution, overlapping of dye luminescence band on long-wave edge of fiber luminescence band occurs. In connection with this, dyed fibers luminesce in a broader spectral band than dye solutions. As concentration is increased, luminescence maximum of dyed fibers shifts from blue to red spectral region. Fibers treated with composition containing $0.5 \mathrm{~g}$ of dye per $1 \mathrm{I}$ of isopropyl alcohol solution have maximal luminescence intensity.

Dependence of luminescence intensity of dye solutions and fibers treated by them is of nonlinear character (Figure 7). The higher dye concentration is in the solution, the larger number of dye molecules is absorbed in superficial layer of fiber (curve 2). Concentration quenching of luminescence of fibers begins happening at their treatment by solutions with dye concentration exceeding $0.5 \mathrm{~g} / \mathrm{l}$. Optimal concentration of dye in the solution for dying fibers on the assumption of data of Figure 7 should be considered $0.4 \div 0.5 \mathrm{~g}$ of dye per $1 \mathrm{I}$ of solution.

Luminescence spectra of fibers modified by different kinds of dyes are shown in Figure 8. Luminescence intensity of these fibers increases in the range of

\section{Phenalemine $\rightarrow$ Fluorescein $\rightarrow$ Eosin $\rightarrow$ Rhodamine $\rightarrow$ Oxazine}

while luminescence intensity of used solutions grows in another order:

$\underset{\rightarrow \text { Rhodamine } \rightarrow \text { Eosin. }}{\rightarrow}$ Fluorescein $\rightarrow$ Oxazine




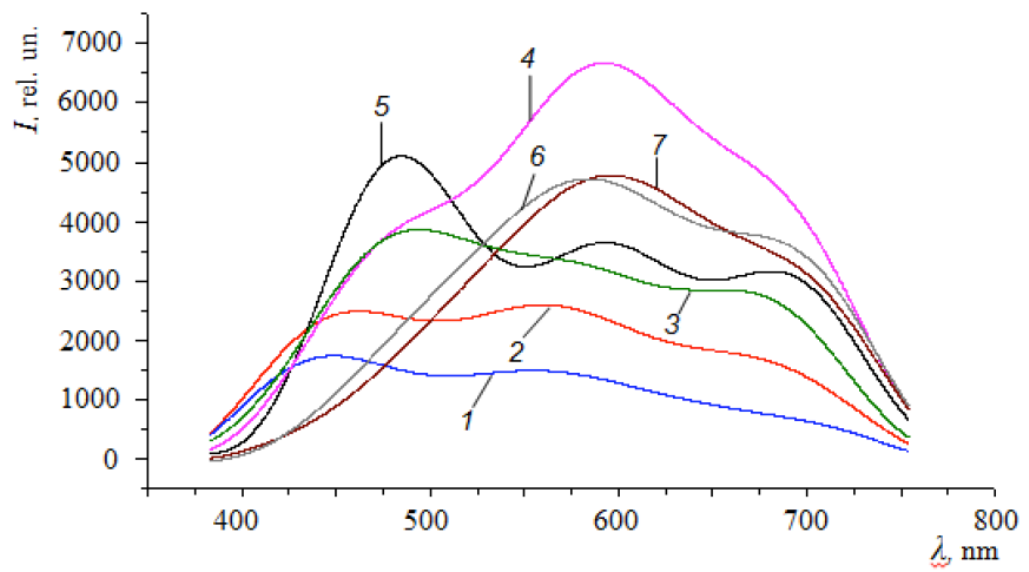

Figure 6: Luminescence of fibers treated by Rhodamine solutions in propanol at dye concentration: $1-0.05 \mathrm{~g} / ; ; 2-0.1 \mathrm{~g} / ; 33-$ $0.2 \mathrm{~g} / \mathrm{l} ; 4-0.5 \mathrm{~g} / \mathrm{l} ; 5-1 \mathrm{~g} / \mathrm{l} ; 6-2 \mathrm{~g} / \mathrm{l} ; 7-4 \mathrm{~g} / \mathrm{l}$.

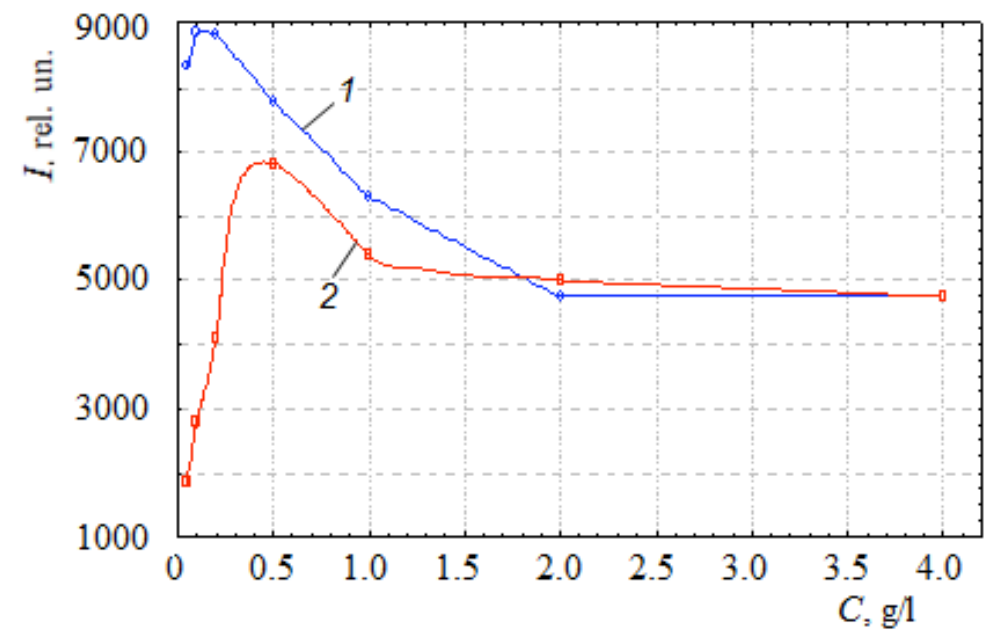

Figure 7: Dependence of luminescence brightness on dye concentration: 1 - in solutions; 2 - in dyed fibers.

One can assume that overlapping of dye solutions spectra on luminescence spectrum of polyester fiber leads to luminescence maximums shifting to red spectral region, luminescence band widening.

Fibers treated by Oxazine and Rhodamine are characterized by the same color and glow in UV light, only pink tints are different. Peculiarity of fibers treated by Fluorescein and Eosin is in the fact that these fibers' colors are different at daylight and exposure to UV radiation. In our opinion, both types of fibers can be employed for protection of documents from counterfeit together or in combination with other types of protective fibers as the standard requirements regulate the necessity to incorporate not less than two varieties into paper pulp.

Fibers modified by Rhodamine (Figure 8, curve 1) have luminescence maximums in red, purple and blue ranges of spectral regions which stipulate bright orange-pink color of glow.
Fibers dyed by Phenalemine (curve 2) have the least intensity of glow, it being complicated to distinguish clear maximums corresponding to any particular spectrum section. In UV light they have pale lilac-pink coloration.

Fibers treated by Oxazine (curve 3) have luminescence maximum approximately in the same region as fibers modified by Rhodamine. Luminescence intensity of these fibers is the highest as compared to other samples, the color of glow is reddish-pink.

Fluorescein (curve 4) gives luminescence maximums in yellow and blue ranges of spectral regions which stipulate yellow-green color of glow. Eosin (curve 5) gives to fibers dim pinky-orange color of glow in UV rays.

Out of examined variants fibers dyed by Phenalemine are less efficient for protection 


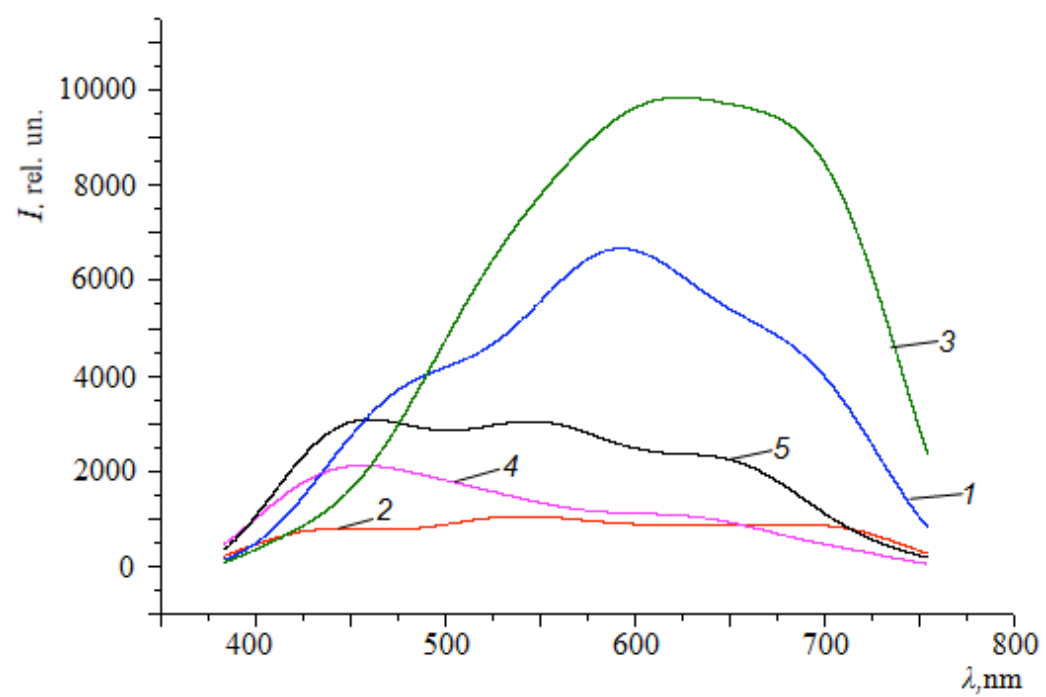

Figure 8: Luminescence of fibers treated by dye solutions in propanol at dye concentration: 0.5 g/l: 1 - Rhodamine; 2 Phenalemine; 3-Oxazine; 4 - Fluorescein; 5-Eosin.

documents from forgery since they are distinguished by low intensity of luminescence compared to other fibers. More reliable protection of documents will be provided by fibers treated by other kinds of dyes especially by Oxazine and Rhodamine which supply intensive luminescence of fiber in UV radiation.

\section{CONCLUSIONS}

Effective method of solving the problem of securities protection from counterfeiting is to incorporate dyed chemical fibers into paper pulp composition. Protective fibers containing luminescent coloring agents are effective, however, fixing them on fiber surface represents a sophisticated technological problem. Currently, the most promising method of modifying protective fibers is to incorporate into them target additives by crazing mechanism. This highperformance processing technique allows combining the process of fibers stretching with incorporating any complex of modifying additives into them. When sealed in crazes of fiber, micro inclusions of target additives do not undergo the effect of alkaline paper pulp which "extinguishes" luminescence and impairs other properties of protective fibers.

Method of fibers intermittent modifying in the process of orientation stretching and design of the apparatus to obtain fibers with alternation of luminescent dyed and undyed areas of set length have been developed. Technologies of modifying chemical fibers by crazing mechanism allow implementing measured incorporation of target additives on local sections of fiber, thus satisfying the requirements of criteria for protection of securities filled with such fibers papers from forgery in the optimal way.

It has been demonstrated that intensity and glow color of fibers significantly depend on kind and concentration of dye in modifying solution. Fibers with Oxazine dye have shown the highest intensity of glow with bright red color among all types of investigated fibers. They give maximum of luminous intensity on the spectrum of luminescence in the region of wavelength $620 \div 700 \mathrm{~nm}$ which corresponds to red light. Fibers with other investigated dyes have lower luminous intensity and their corresponding intensity maximums are shifted to shorter wave region. Correspondingly the color of their glow is from orange to green.

It has been determined that dye concentration in modifying liquid affects luminous intensity of fibers in UV light and even (to some extent) - the color of glow. It has been shown with Rhodamine example that fibers modified by solution with dye content $0.5 \mathrm{~g} / \mathrm{l}$ have the highest luminous intensity (brightness). The reduction of dye concentration results, in the first place, in sharp decrease of luminous intensity of fibers and, secondly, in some shifting of intensity maximum on the spectrum to shorter wavelength, i.d. from bright red to yellow and yellow-green. The increase of dye concentration higher $0.5 \mathrm{~g} / \mathrm{l}$ practically does not influence the colour of fibers glow but slightly decreases its intensity.

\section{REFERENCES}

[1] Belousov AG. Comprehensive criminalistic investigation of securities and banknotes protection facilities. Moscow: Economical Center of Ministry of Internal Affairs of Russia 2001. 
[2] Kislukhin C, Korochkin L. Protection of official papers, forms and documents from forgery in the Republic of Belarus. Bankauski vesnik (Belarus) 2002; 6: 6-40.

[3] Korochkin L, Gorelenko A. Problems of protection of banknotes and securities from forgery, Bankauski vesnik (Belarus) 2002; 9: 42-5.

[4] Konshin AA. Protection of printing production from falsification. Moscow: Sinus Ltd. Co 1999.

[5] Perepyolkin KE. Principles and methods of modifying fibers and fiber materials. Chem Fibers (Russia) 2005; 2: 37-49.

[6] Razd'akonova GI, Surovkin VF, inventors; Technical Carbon Engineering and Design Institute of Siberian Division of Russian Academy of Sciences, assignee. Composition for chemical fibers dyeing in the main. Russian patent $R U$ 21300971999.

[7] Nagorny VS, Levchenko YuA, inventors; Nagorny VS, Levchenko YuA, assignee. Process of Fibers Dyeing. Russian patent RU 21441032000.

[8] Kim D-K, Park Y-H, inventors; Korea Security Printing and Minting Co., assignee. Process of Manufacturing Fibers protected from Falsification. Russian patent RU 2190716 2002.

[9] Peisakhovich AA, Pavutnitski VV. Vapor-phase method of dyeing synthetic sewing threads and yarns. Textile Industry (Russia) 2010; 4: 34-6.
[10] Goldade VA, Pinchuk LS, Vinidiktova NS. Modification of polyester fibers by bactericides using crazing mechanism. Int Polym Proc 2010; 25: 199-204. http://dx.doi.org/10.3139/217.2321

[11] Kramer EJ, Berger LL. Fundamental processes of craze growth and fracture. Adv Polym Sci 1990; 91/92: 61-8. http://dx.doi.org/10.1007/BFb0018018

[12] Volynski AL. Rehbinder's effect in polymers. Priroda (Russia) 2006; 11: 11-8.

[13] Volynski AL, Bakeev NF. Structural self-organization of amorphous polymers. Moscow: Physmatlit 2005.

[14] Volynski AL, Mikushev AE, Jarysheva LM, Bakeev NF. Crazing in liquid media - a basis for generation unique method of modifying polymers. Russ Chem J 2005; 49(6): 118-28.

[15] Vinidictova NS, Pinchuk LS, Goldade VA, Novikov VP. Application of polymer crazing for creation antimicrobial polyester fibers. Reports of Belarus NAS 2009; 53 (5): 10812.

[16] Andronova AP, Poprjadukhina SI, Egorov IA, Aljoshicheva NB. Dyeing of polyester fibers by using crazing method. Chem Fibers (Russia) 2011; 1: 71-4.

[17] Pinchuk LS, Kuzmenkova NV, Goldade VA, et al, inventors; V.A. Belyi Metal-Polymer Research Inst of NAS of Belarus, assignee. Arrangement for discontinuous coloration of chemical fibers. Belarus patent BY 183642014. 